\title{
Electrokinetic behavior of two touching inhomogeneous biological cells and colloidal particles: Effects of multipolar interactions
}

\author{
J. P. Huang \\ Department of Physics, The Chinese University of Hong Kong, Shatin, NT, Hong Kong, and \\ Biophysics and Statistical Mechanics Group, Laboratory of Computational Engineering, \\ Helsinki University of Technology, P. O. Box 9203, FIN-02015 HUT, Finland \\ Mikko Karttunen \\ Biophysics and Statistical Mechanics Group, Laboratory of Computational Engineering, \\ Helsinki University of Technology, P. O. Box 9203, FIN-02015 HUT, Finland \\ K. W. Yu and L. Dong \\ Department of Physics, The Chinese University of Hong Kong, Shatin, NT, Hong Kong \\ G. Q. $\mathrm{Gu}$ \\ Department of Physics, The Chinese University of Hong Kong, Shatin, \\ NT, Hong Kong, and College of Information Science and Technology, \\ East China Normal University, Shanghai 200 062, China
}

\begin{abstract}
We present a theory to investigate electro-kinetic behavior, namely, electrorotation and dielectrophoresis under alternating current (AC) applied fields for a pair of touching inhomogeneous colloidal particles and biological cells. These inhomogeneous particles are treated as graded ones with physically motivated model dielectric and conductivity profiles. The mutual polarization interaction between the particles yields a change in their respective dipole moments, and hence in the AC electrokinetic spectra. The multipolar interactions between polarized particles are accurately captured by the multiple images method. In the point-dipole limit, our theory reproduces the known results. We find that the multipolar interactions as well as the spatial fluctuations inside the particles can affect the AC electrokinetic spectra significantly.

PACS numbers: 82.70.-y,77.22.Gm,77.22.-d,77.84.Lf
\end{abstract}

\section{INTRODUCTION}

Identification and analysis of cell populations and (micro)biological particles are essential in many practical applications ranging from cancer research to chemical analysis of environmental pollutants. During the past decade, alternating current (AC) electrokinetic phenomena, and in particular electrorotation (ER) and dielectrophoresis (DEP), have received much attention in this respect, especially in micromanipulation and separation of submicron size particles [1, 2, 3, 4, 5, 6, 7, 8, 9, 10, 11, 12]. In addition to biological and environmental applications, AC electrokinetic phenomena have been suggested as possible mechanisms for nanomotors [13, 14].

Both dielectrophoresis and electrorotation are based on dielectric properties of particles. These properties depend heavily on the nature of the surface, e.g., size, shape, and charge density. For example, since the composition and shape of cancer cells differ from those of healthy cells, these difference are reflected in their characteristic dielectric properties which can be exploited in identifying them. From a practical point of view, AC electrokinetic methods have the advantages of short detection times and high sensitivity [5].

Dielectrophoresis can be defined as the movement of polarizable particles in a non-uniform applied AC electric field [15], whereas in electrorotation an interaction between a rotating AC electric field [6] and suspended particles leads to a rotational motion of the particles. The most commonly used models to deal with the dielectric properties of colloidal particles or biological cells are the so called shell models. Because of inhomogeneous compartmentalization of biological cells, one-, two-, and three- shell models have been applied to discuss electrorotation of biological cells, e.g., see Refs. [6, 16, 17].

These cell models have several limitations and they become complex as the number of shells increases. This is particularly true when two (or more) particles approach each other. In the dilute limit, one can focus on the electrokinetic spectra of an individual particle. If the suspension is not dilute, as it is often the case in practice, the situation is complicated by the existence of multipolar interactions. Even when a suspension is initially in the dilute limit, particles often aggregate due to the presence of an external electric field. In this case, a point dipole approximation [18, 19] becomes inadequate and the mutual interactions must be taken into account by a theory [20, 21] that goes beyond the point dipole.

To provide a physically motivated and tractable model for inhomogeneous particles, such as cells, we have recently studied particles with spatial gradients in their structures by introducing profiles for the conductivities and dielectric constants of the particles, and used differential approximation for the dielectric factor [22, 23, 24]]. Here, we extend this work to take into account polarization interactions when two particles approach each other, and treat both DEP and ER using the same theoretical framework. We consider a pair of touching graded particles in suspensions. As a result, the mutual polarization 


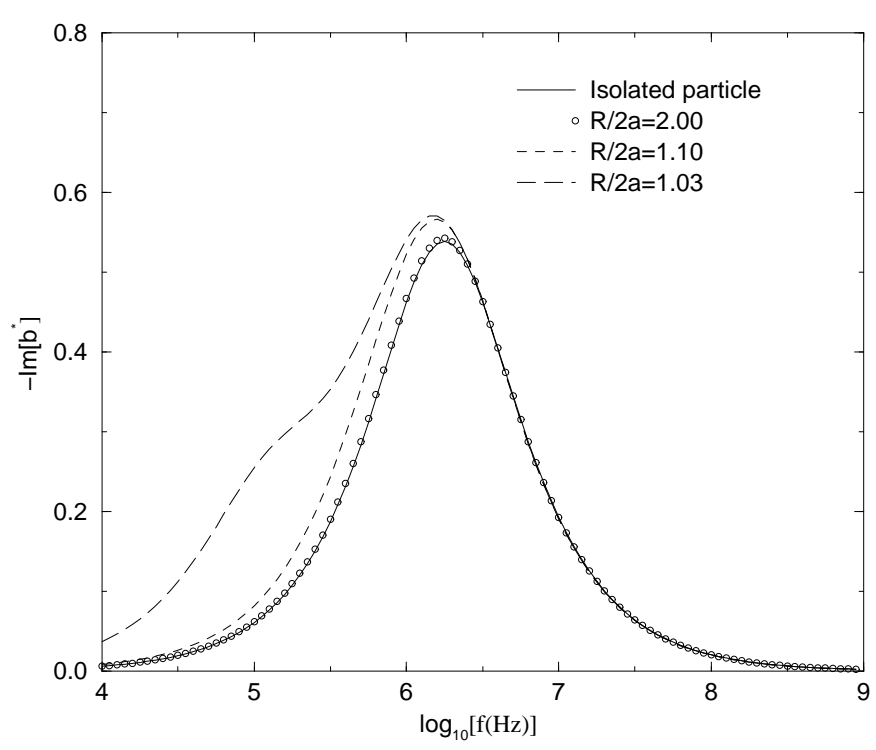

FIG. 1: ER spectra for an isolated particle and two touching particles with $R / 2 a=2.00,1.10,1.03$, respectively. Parameters: $c=-30 \epsilon_{0}, m=1.0$.

interactions and the gradation fluctuations inside the particles lead to significant changes in the electro-kinetic spectra. In a more general context, these are manifestations of correlation effects in charge-carrying system [25, 26].

\section{FORMALISM}

We consider inhomogeneous biological cells or colloidal particles with radius $a$. We assume that they have a distancedependent complex dielectric constant $\tilde{\epsilon}_{1}(r)(0<r \leq a)$, and that they are embedded in a host fluid having dielectric constant $\tilde{\epsilon}_{2}$. Here $\tilde{\epsilon}=\epsilon(r)+\sigma(r) /(2 \pi i f)$, where $\epsilon$ denotes the real dielectric constant, $\sigma(r)$ the conductivity, $f$ the frequency of an external field, and $i \equiv \sqrt{-1}$. As the above formulas suggest, $\epsilon(r)$ and $\sigma(r)$ are not constant inside the particle but have distance dependent profiles. This is a very physical assumption and we will return to it in the following discussion and later in connection with the numerical simulations.

The dipole, or Clausius-Mossotti, factor reflects the polarization of a particle in a surrounding medium. In a recent work [22], we derived the dipole factor for graded spherical particles by introducing a differential effective dipole approximation (DEDA). The generalization to the nonspherical case was done as well [23]. The idea of the DEDA can be summarized as follows: Consider a shell model for an inhomogeneous particle. In the DEDA one adds new shells of infinitesimal thickness to the particle. Each of these cells have distance-dependent complex dielectric constant. Since the thickness of the layer approaches zero $(\mathrm{d} r \rightarrow 0)$, its correction to the dipole factor is infinitesimal and one could eventually obtain a differential equation.
The DEDA equation for a spherical graded particle has the form [22, 23]

$$
\begin{gathered}
\frac{\mathrm{d} b}{\mathrm{~d} r}=-\frac{1}{3 r \tilde{\epsilon}_{2} \tilde{\epsilon}_{1}(r)}\left[(1+2 b) \tilde{\epsilon}_{2}-(1-b) \tilde{\epsilon}_{1}(r)\right] \\
{\left[(1+2 b) \tilde{\epsilon}_{2}+2(1-b) \tilde{\epsilon}_{1}(r)\right]}
\end{gathered}
$$

It is worth noting that the DEDA is essentially exact since it is in an excellent agreement with the exact solutions obtained for a power-law profile and a linear profile by solving the Laplace equation for the local electric field [24].

For a pair of particles at a separation $R$ in a suspension, we have to consider the multiple images effect [20, 27]. Theoretically, we may see the inhomogeneous graded particle as an effectively homogeneous one. Then, we consider two particles in a suspension which is subject to an external uniform electric field. This yields a dipole moment into each particle. Let us denote the dipole moments of particles 1 and 2 as $p_{10}$ and $p_{20}$ ( $\equiv p_{10}$ for identical particles), respectively. Then, we take into account the image effects. The dipole moment $p_{10}$ induces an image dipole $p_{11}$ into particle 2 , while $p_{11}$ induces another image dipole in particle 1 . As a result, multiple images are formed. The same description holds for $p_{20}$. Thus, we admit the infinite series of image dipoles. To this end, we obtain the sum of dipole moments inside each particle, and derive the desired expressions for dipole factors. Let us consider two basic cases: 1) longitudinal field (L), where the field is parallel to the line joining the centers of the particles, and 2) transverse field (T), where the field is perpendicular to the line joining the centers of the particles.

Based upon a multiple images method [27], the dipole factors, $b_{L}{ }^{*}$ and $b_{T}{ }^{*}$, are given by [20, 27]

$$
\begin{aligned}
& b_{L}^{*}=b \sum_{n=0}^{\infty}(2 b)^{n}\left(\frac{\sinh \alpha}{\sinh (n+1) \alpha}\right)^{3}, \\
& b_{T}^{*}=b \sum_{n=0}^{\infty}(-b)^{n}\left(\frac{\sinh \alpha}{\sinh (n+1) \alpha}\right)^{3}
\end{aligned}
$$

for longitudinal and transverse field cases, respectively, where $\alpha$ satisfies the relation $\cosh \alpha=R / 2 a$. Although it is not obvious, it is important to notice that multipoles are included in the above formulas [28]. Clearly, the multiple images effects have been taken into account in $b_{L}^{*}$ and $b_{T}^{*}$. It is worth noting that: Setting $n$ up to 1 in the two equations leads to the dipole factor for two touching particles in the point-dipole limit. In this case, in view of both $|b|^{2} \ll 1$ and $R / 2 a \sim 1$, we have

$$
\begin{aligned}
& b_{L}^{*}(1)=\frac{b}{1-b / 4} \\
& b_{T}^{*}(1)=\frac{b}{1+b / 8}
\end{aligned}
$$

Both Eqs.(4) agree well with the result of Jones, which were obtained by a field method in the point-dipole limit [19]. 

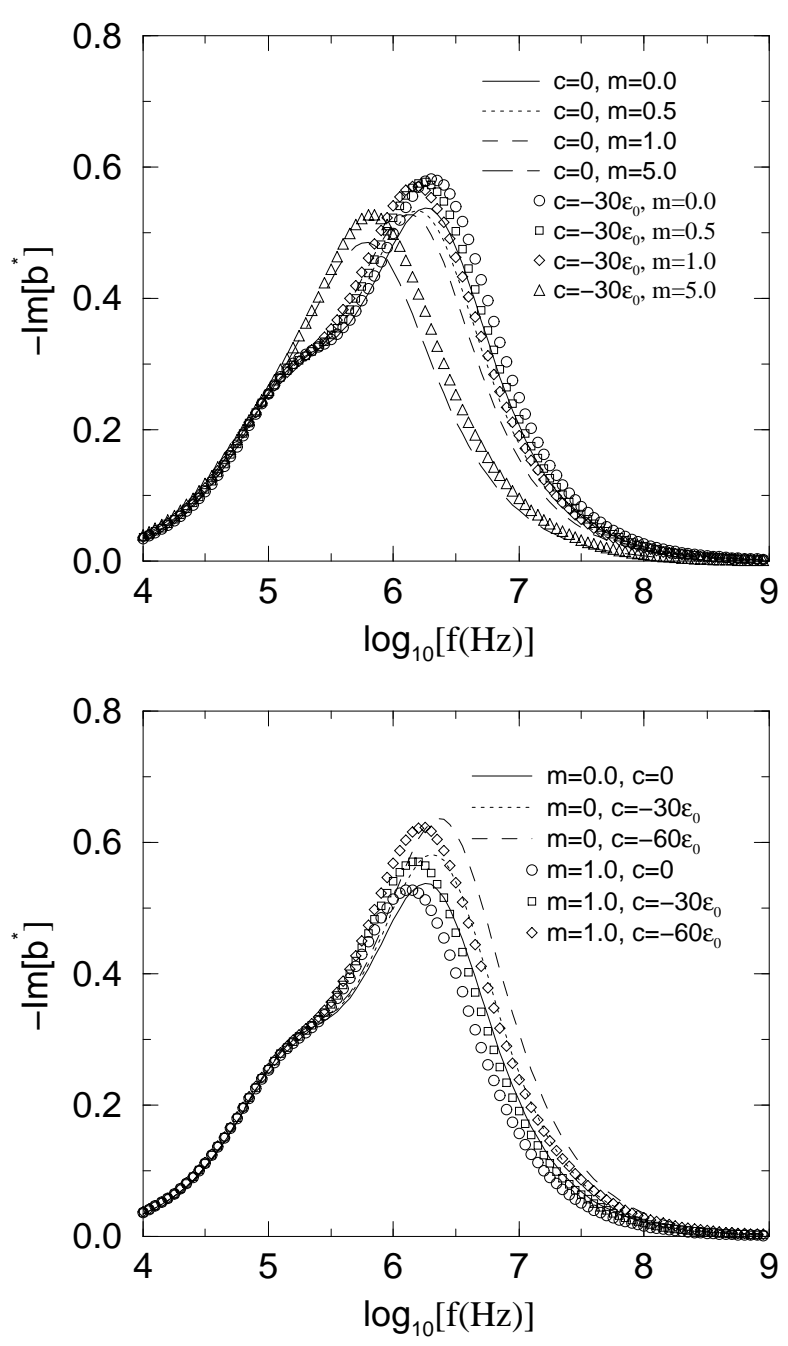

FIG. 2: ER spectra for two touching particles. Upper panel for profile constants $m$ at $c=0$ and $c=-30 \epsilon_{0}$, respectively. Lower panel for various $c$ at $m=0$ and $m=1.0$, respectively. The spectrum is given as the imaginary part of the dipole factor. Parameter: $\mathrm{R} / 2 \mathrm{a}=1.03$.

\section{A. Electrorotation}

By adding a rotating electric field with magnitude $E_{\mathrm{ER}}{ }^{*}$ to the two particle system, the effective dipole factor for a pair of particles should be given by [20]

$$
b^{*}=\left(b_{L}{ }^{*}+b_{T}^{*}\right) / 2 .
$$

Thus, in this case, the electrorotation velocity of a particle $\Omega^{*}$ is given by [20]

$$
\Omega^{*}=-\phi\left(\epsilon_{2}, \eta_{2}, E_{\mathrm{ER}}^{*}\right) \operatorname{Im}\left[b^{*}\right],
$$

where $\phi\left(\epsilon_{2}, \eta_{2}, E_{\mathrm{ER}}^{*}\right)$ is a function of $\epsilon_{2}$, the viscosity of the medium $\eta_{2}$, and $E_{\mathrm{ER}}{ }^{*}$. Here $\operatorname{Im}[\cdots]$ denotes the imaginary part of $[\cdots]$. For an isolated spherical particle, $\phi\left(\epsilon_{2}, \eta_{2}, E_{\mathrm{ER}}{ }^{*}\right)=\epsilon_{2} E_{\mathrm{ER}}{ }^{* 2} / 2 \eta_{2}[29]$.
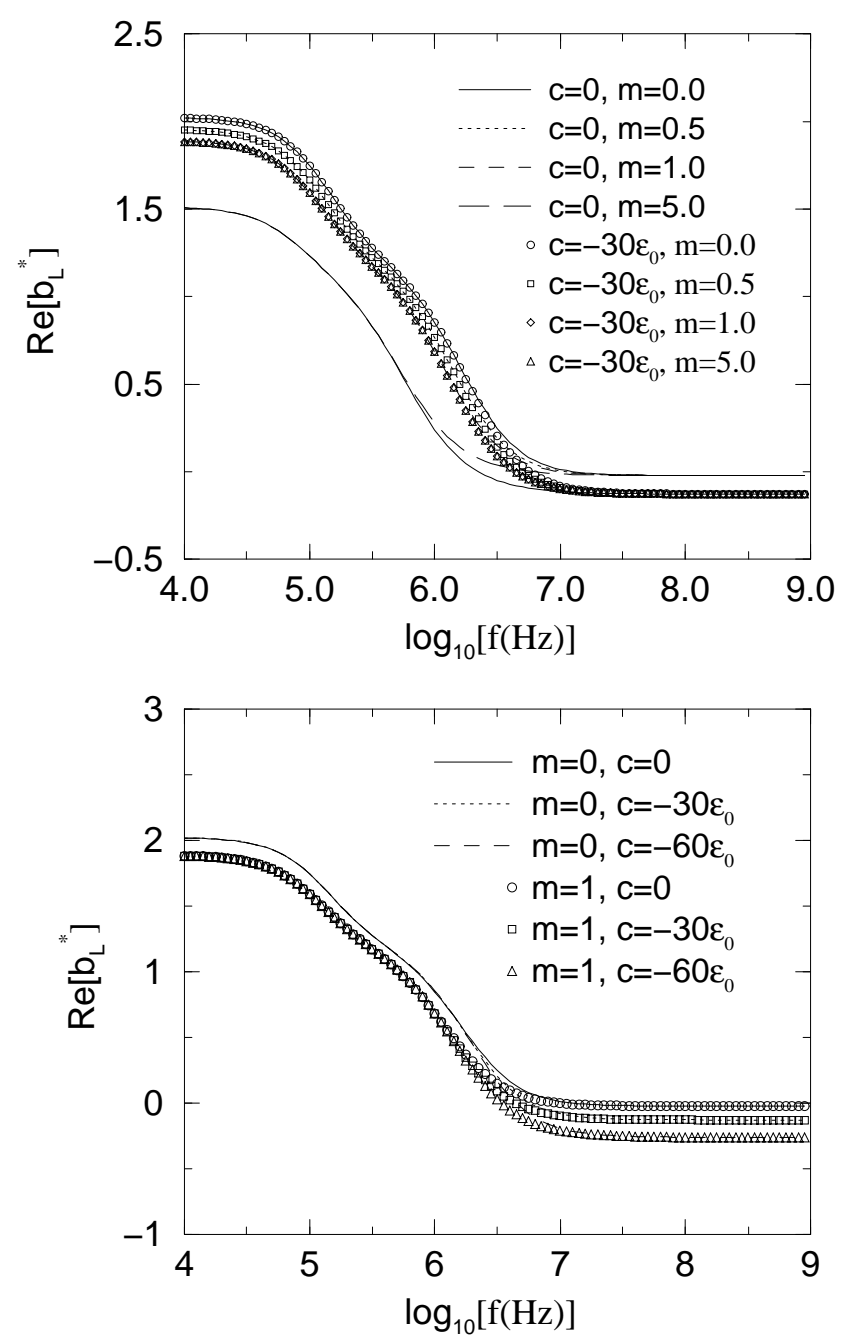

FIG. 3: DEP spectra for two touching particles in the longitudinal field case. Upper panel for profile constants $m$ at $c=0$ and $c=$ $-30 \epsilon_{0}$, respectively. Lower panel for various $c$ at $m=0$ and $m=$ 1.0, respectively. The spectrum is given as the real part of the dipole factor. Parameter: $\mathrm{R} / 2 \mathrm{a}=1.03$.

\section{B. Dielectrophoresis}

We consider a single particle suspended in a medium and subjected to a nonuniform AC electric field $\mathbf{E}_{\mathrm{DEP}}{ }^{*}$. The DEP force $\mathbf{F}_{\mathrm{DEP}}$ acting on the particle is given by [29]

$$
\mathbf{F}_{\mathrm{DEP}}=2 \pi \epsilon_{2} a^{3} \operatorname{Re}[b] \nabla\left|\mathbf{E}_{\mathrm{DEP}}{ }^{*}\right|^{2},
$$

where $\mathbf{E}_{\mathrm{DEP}}{ }^{*}$ stands for the local RMS electric field, and $\operatorname{Re}[\cdots]$ denotes the real part of $[\cdots]$. Next, for a pair of touching particles, the DEP force is given by [21, 29]

$$
\begin{aligned}
& \mathbf{F}_{L}{ }^{*}=2 \pi \epsilon_{2} a^{3} \operatorname{Re}\left[b_{L}{ }^{*}\right] \nabla\left|\mathbf{E}_{\mathrm{DEP}}{ }^{*}\right|^{2}, \\
& \mathbf{F}_{T}{ }^{*}=2 \pi \epsilon_{2} a^{3} \operatorname{Re}\left[b_{T}{ }^{*}\right] \nabla\left|\mathbf{E}_{\mathrm{DEP}}\right|^{2},
\end{aligned}
$$

for longitudinal and transverse field cases, respectively. The above formulation for the DEP force is, stricly speaking, ap- 

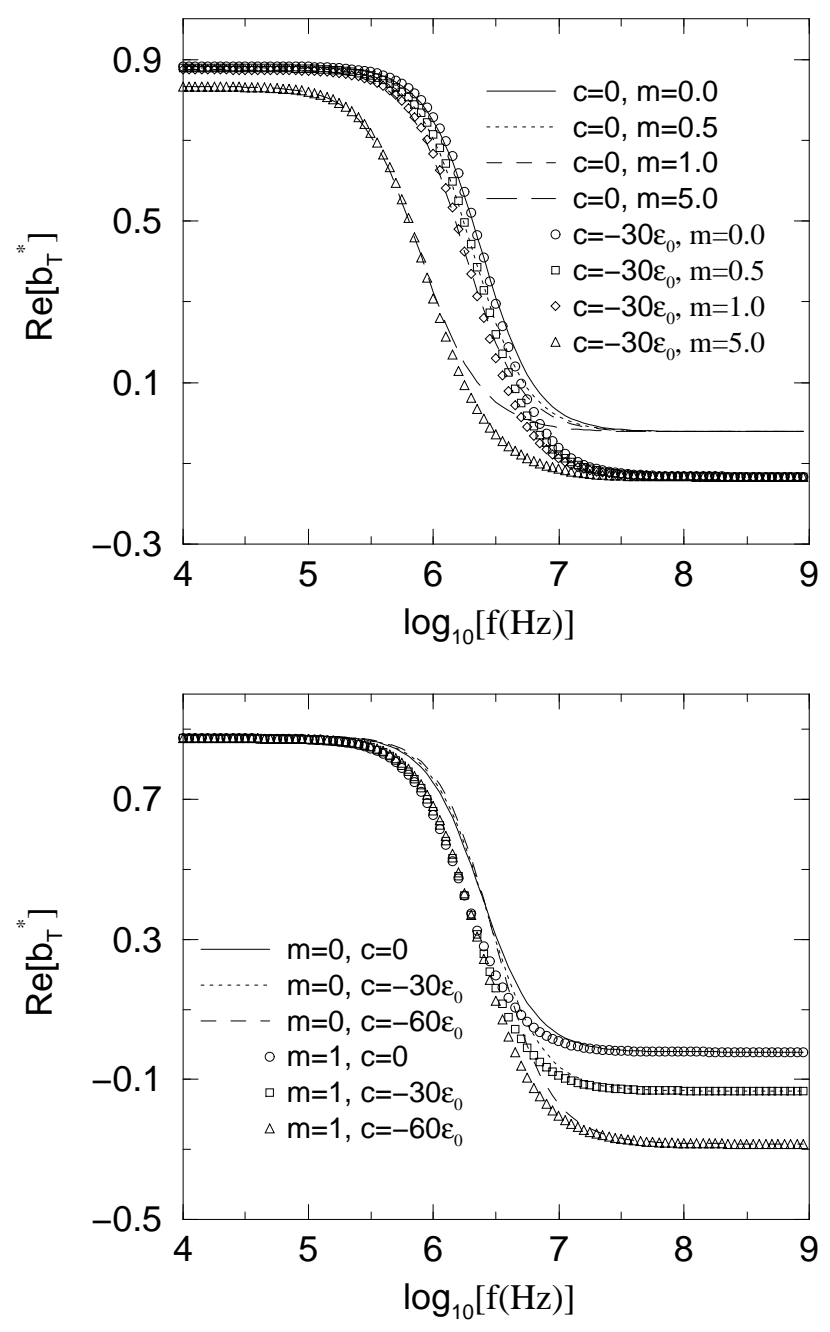

FIG. 4: Same as in Fig. 3 but in the transverse field case.

plicable for linearly polarized fields [30], which is the case studied here.

\section{NUMERICAL RESULTS}

For the following numerical calculations, we take the conductivity and dielectric profiles to be

$$
\begin{array}{ll}
\sigma_{1}(r)=\sigma_{1}(0)(r / a)^{m}, & r \leq a \\
\epsilon_{1}(r)=\epsilon_{1}(0)+c(r / a), & r \leq a
\end{array}
$$

where $m$ and $c$ are profile dependent constants. The profile is clearly physical since conductivity can change rapidly near the boundary of cell and a power-law profile prevails [23]. On the other hand, the dielectric constant may vary only slightly and thus a linear profile suffices [23]. In particular, the dielectric constant at the center, namely $\epsilon_{1}(0)$, may be larger than that at the boundary. Thus, in what follows, we would choose $c \leq 0$. By integrating the dielectric profile, we obtain an average dielectric constant $\epsilon_{a v}$ for different values of $c$ by using a
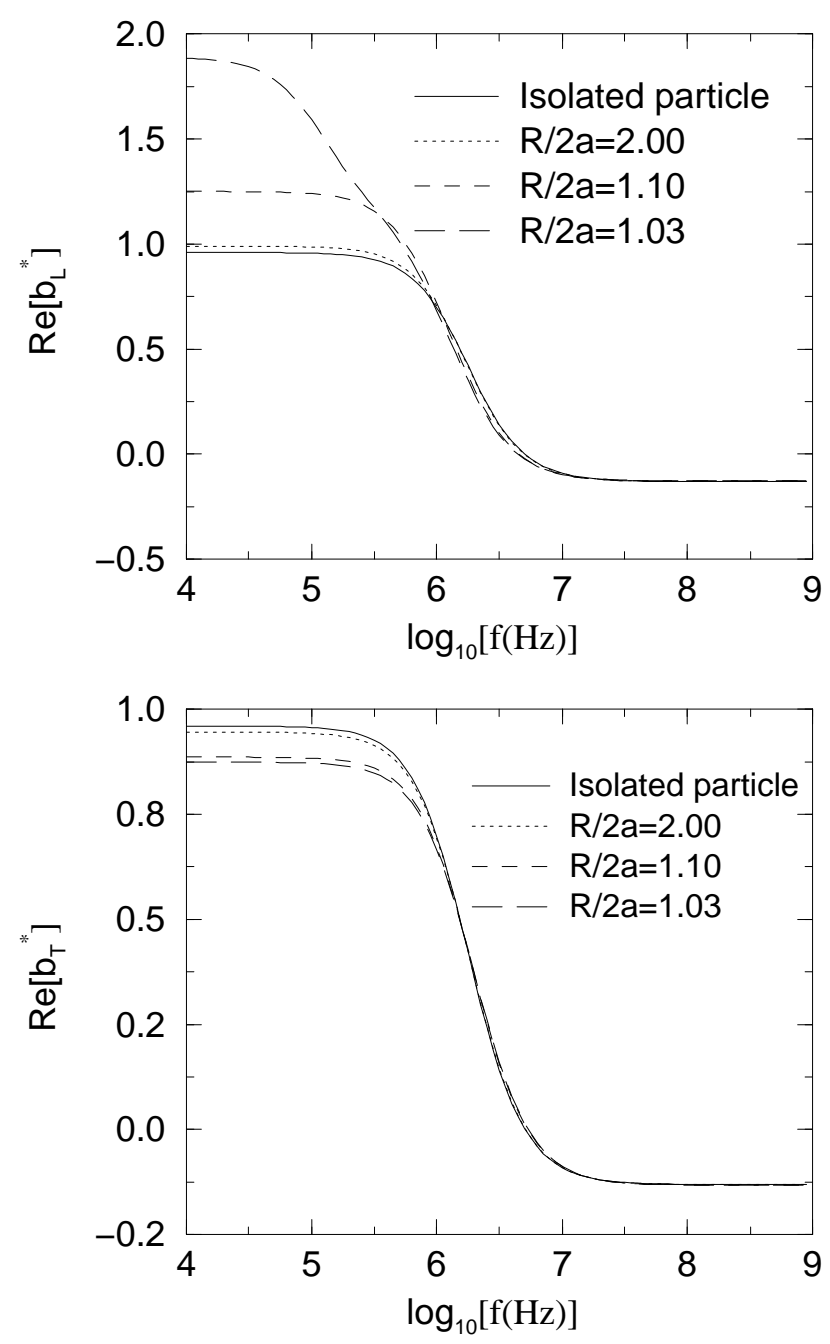

FIG. 5: DEP spectra in the longitudinal field case (upper panel) and the transverse (lower panel), for an isolated particle, and two touching particles with separation ratio $R / 2 a=2.00,1.10,1.03$, respectively. Parameters: $c=-30 \epsilon_{0}, m=1.0$.

volume average [23]

$$
\epsilon_{1}^{a v}=\frac{\int_{0}^{a} \epsilon_{1}(r) r^{2} \mathrm{~d} r}{\int_{0}^{a} r^{2} \mathrm{~d} r}
$$

For the above dielectric profile $\epsilon_{1}^{a v}=\epsilon_{1}(0)+3 c / 4$.

For all numerical calculations, we take $\epsilon_{1}(0)=75 \epsilon_{0}, \epsilon_{2}=$ $80 \epsilon_{0}, \sigma_{1}(0)=2.8 \times 10^{-2} \mathrm{~S} / \mathrm{m}$, and $\sigma_{2}=2.8 \times 10^{-4} \mathrm{~S} / \mathrm{m}$. Here $\epsilon_{0}$ denotes the dielectric constant of the free space.

Figure 11 shows the ER spectrum of two particles at different distances from each other. At large separations (e.g. $R / 2 a>2$ ), the multipolar interaction may be neglected, but the induced multiple images play an important role in the spectrum when two particles approach each other.

In Fig. 2] it is evident that a second peak due to the multiple image effect occurs at a lower frequency. In fact, the appearance of a second peak has been predicted for homogeneous particles in a recent work [20]. Moreover, fluctuations 
in the conductivity profile can make the characteristic frequency shifted to lower frequencies (red-shifted), while those in the dielectric profile can enhance the peak value. However, such effects on the second characteristic frequency and its peak value are small enough to be neglected.

Fluctuations in conductivity and dielectric profiles may enhance the DEP spectrum not only in the longitudinal field case (see Fig 3), but also in the transverse (see Fig 4). The effects of multiple images may change the DEP spectrum significantly.

Similar to Fig. 11 Figure 5 shows that the multiple images play a crucial role in the DEP spectrum when the particles are close enough. In contrast to the result from an isolated particle, the multiple image effect may enhance the DEP spectra (moreover, the real part of the dipole factor may be enhanced to be larger than 1) at low frequency region in the longitudinal field case. However, the DEP spectrum is reduced in the transverse field case due to the presence of multiple images.

In addition, we have also compared the point dipole model with the current multiple image dipole model (no figures shown). As expected, the results predicted by them are quite different, especially at low frequencies. This shows further that the point dipole model is inadequate for the touching particles, and thus needs to be modified to take into account the effect of multiple images.

\section{DISCUSSION AND CONCLUSION}

In this work, we investigated the effects of multipolar interactions on $\mathrm{AC}$ electrokinetic behavior, electrorotation and dielectrophoresis, of inhomogeneous biological cells and colloidal particles. We model such inhomogeneous particles as graded ones. Our method may be extended to high concentration case [31, 32] or pearl chain case [33, 34], work is in progress to address these issues in detail. Also, it is possible to take into account shape effects by considering the nonspherical shapes, such as oblate or prolate spheroid [11]. In doing so, we might resort to the derived DEDA equation for graded spheroidal particles as well [23]. It is also straightforward to extend this work to deal with the experimentally interesting case of charged colloidal suspensions [21].

To put our approach in the context of composite particles, we have performed a mean-field approach in the spirit of Choy et al. [35, 36], i.e., treating inhomogeneous particles as effectively homogeneous ones which are embedded in a uniform field. In particular, it is worth noting that well-known Tartar formula [37] can be used to exactly calculate the effective complex dielectric constant of a single graded particle. Thus, once this effective complex dielectric constant is obtained, one can proceed to calculate the relevant dipole moment, and hence the desired dipole factor. More interestingly, our DEDA approach can predict exactly the same result as Tartar formula [38].

To sum up, based on the DEDA, we have presented a theoretical study of electrokinetic behavior, electrorotation (ER) and dielectrophoresis (DEP) for two touching inhomogeneous particles in suspensions. We found mutual polarization effects and the spatial fluctuations inside colloidal particles or biological cells can both affect ER and DEP spectra significantly. Our approach has the further advantage of being able to treat both electrorotation and dielectrophoresis using the same theoretical framework.

As a further study and a test to our theory, it would be interesting to have a systematic experimental investigations of these effects. The hope is that they would shed light to the limits of the theory and that they would help to separate the DEP and ER behavior from, e.g. electrohydrodynamic flow effects [39] and limitations due to Brownian motion. One possibility for doing so would be to use the laser tweezers combined with ER and/or DEP [39, 40].

\section{Acknowledgments}

This work has been supported by the Research Grants Council of the Hong Kong SAR Government under project number CUHK 4245/01P, and by the Academy of Finland (M.K.) and the Finnish Academy of Science and Letters (M. K.). One of us (M. K.) would like to thank Andreas Manz for inspiring discussions.
[1] X. Duan, Y. Huang, Y. Cui, J. Wang, and C. M. Lieber, Nature 409, 66 (2001).

[2] M. P. Hughes, H. Morgan, F. J. Rixon, J. P. H. Burt, and R. Pethig, Biochim. Biophys. Acta 1425, 119 (1998).

[3] H. Morgan, M. P. Hughes, and N. G. Green, Biophys. J. 77, 516 (1999).

[4] C. F. Chou, J. O. Tegenfeldt, O. Bakajin, S. S. Chan, E. C. Cox, N. Darnton, T. Duke, and R. H. Austin, Biophys. J. 83, 2170 (2002).

[5] K. Ratanachoo, P. R. C. Gascoyne, and M. Ruchirawat, Biochim. Biophys. Acta 1564, 449 (2002).

[6] W. M. Arnold and U. Zimmermann, Z. Naturhorsch. 37c, 908 (1982).

[7] G. Fuhr, U. Zimmermann, and S. Shirley, in Electromanipulation of cells, edited by U. Zimmermann and G. A. Neil (CRC
Press, Boca Raton, FL, 1996), pp. 259-328.

[8] J. Yang, Y. Huang, X. Wang, X. B. Wang, F. Becker, and P. R. C. Gascoyne, Biophys. J. 76, 3307 (1999).

[9] F. F. Becker, X. B. Wang, Y. Huang, R. Pethig, J. Vykoukal, and P. R. C. Gascoyne, Proc. Natl. Acad. Sci. USA 92, 860 (1995).

[10] K. L. Chan, H. Morgan, E. Morgan, I. T. Cameron, and M. R. Thomas, Biochim. Biophys. Acta 1500, 313 (2000).

[11] J. P. Huang and K. W. Yu, J. Phys.: Condens. Matter 11, 1213 (2002).

[12] P. R. C. Gascoyne and J. Vykoukal, Electrophoresis 23, 1973 (2002).

[13] M. P. Hughes, Nanotechnology 11, 124 (2000).

[14] M. P. Hughes, Nanotechnology 13, 157 (2002).

[15] H. A. Pohl, Dielectrophoresis (Cambridge Univ. Press, Cambridge, 1978). 
[16] G. Fuhr and P. I. Kuzmin, Biophys. J. 50, 789 (1986).

[17] K. L. Chan, P. R. C. Gascoyne, F. F. Becker, and R. Pethig, Biochim. Biophys. Acta 1349, 182 (1997).

[18] F. A. Sauer, in Coherent excitations in biological systems, edited by H. Froehlich and F. Kremer (Springer-Verlag, Berlin, 1983), pp. 134-144.

[19] T. B. Jones, J. Electrostatics 25, 231 (1990).

[20] J. P. Huang, K. W. Yu, and G. Q. Gu, Phy. Rev. E 65, 021401 (2002).

[21] J. P. Huang, M. Karttunen, K. W. Yu, and L. Dong, Phys. Rev. E 67, 021403 (2003).

[22] K. W. Yu, G. Q. Gu, and J. P. Huang, cond-mat/0211532 (2002).

[23] J. P. Huang, K. W. Yu, G. Q. Gu, and M. Karttunen, Phys. Rev. E 67, 051405 (2003).

[24] L. Dong, G. Q. Gu, and K. W. Yu, Phys. Rev. B 67, 224205 (2003).

[25] A. Y. Grosberg, T. T. Nguyen, and B. I. Shklovskii, Rev. Mod. Phys. 74, 329 (2002).

[26] M. Patra, M. Patriarca, and M. Karttunen, Phys. Rev. E 67, 031402 (2003).

[27] K. W. Yu and J. T. K. Wan, Comp. Phys. Comm. 129, 177 (2000).

[28] H. Sun and K. W. Yu, Phys. Rev. E 67, 011506 (2003).
[29] T. B. Jones, Electromechanics of particles (Cambridge Univ. Press, Cambridge, 1995).

[30] M. Washizu and T. B. Jones, J. Electrostat. 38, 199 (1996).

[31] J. P. Huang, K. W. Yu, and G. Q. Gu, Phys. Lett. A 300, 385 (2002).

[32] L. Gao, J. P. Huang, and K. W. Yu, Phys. Rev. E 67, 021910 (2003).

[33] J. P. Huang, J. T. K. Wan, C. K. Lo, and K. W. Yu, Phys. Rev. E 64, 061505(R) (2001).

[34] J. P. Huang, L. Gao, and K. W. Yu, J. Appl. Phys. 93, 2871 (2003).

[35] T. C. Choy, A. Alexopoulos, and M. F. Thorpe, Proc. Roy Soc. ser. A 454, 1973 (1998).

[36] T. C. Choy, A. Alexopoulos, and M. F. Thorpe, Proc. Roy Soc. ser. A 454, 1993 (1998).

[37] G. W. Milton, The Theory of Composites (Cambridge University Press, Cambridge, 2002), chap. 7.

[38] L. Gao, J. P. Huang, and K. W. Yu, cond-mat/0308032.

[39] J. Voldman, R. A. Braff, M. Toner, M. L. Gray, and M. A. Schmidt, Biophys. J. 80, 531 (2001).

[40] T. Schnelle, T. Müller, C. Reichle, and G. Fuhr, Appl. Phys. B 70, 267 (2000). 